\title{
CORTO CIRCUITO: PRESERVACIÓN HISTÓRICA Y CULTURAL DE UNA CIUDAD
}

Daniela Agudelo Guisao*, Angie Lizeth Palacios Mena**

\section{Resumen}

Despertó el interés en los estudiantes pertenecientes al programa de Comunicación Audiovisual y Digital y al semillero de investigación, "Memoria y Región" de la Fundación Universitaria del Área Andina, por investigar el fenómeno que surgió a partir del evento cultural pereirano, Corto Circuito, el cual consta de cinco instituciones educativas y culturales en el centro de Pereira, que se unen para ofrecer una noche desbordada de arte, cultura y ciudad.

Inicialmente, se propuso realizar cuatro productos audiovisuales para Corto Circuito, que homenajeen sus 15 años de realización, donde se narre su historia y funcionamiento actual; también, productos de promoción que fortalezcan y aumenten la asistencia. A partir de esta idea, se llevaron a cabo varias entrevistas semiestructuradas a los gestores de cada institución, artistas y a una fundadora, con el fin de obtener información de primera mano de las personas que son la base sólida de este y recopilarla en los productos.

* Estudiante, Comunicación Audiovisual y Digital, Fundación Universitaria del Área Andina. Correo: dagudelo25@estudiantes.areandina.edu.co

* Estudiante, Comunicación Audiovisual y Digital, Fundación Universitaria del Área 


\section{Introducción}

Siempre nos ha preocupado el pasado y el surgimiento de las cosas. Nos gusta sentirnos parte de una comunidad, un grupo. Por eso, en nuestro día a día, nos rodeamos de memoria y debemos remontarnos al pasado para que podamos darnos cuenta de lo que somos o a lo que pertenecemos ahora, dar un sentido a nuestras acciones y reafirmarnos en nosotros. Por tanto, personas de todo el mundo se esfuerzan por proteger las reliquias históricas representadas mediante su patrimonio cultural. Reevaluar el patrimonio tangible del pueblo, aporta tradición. Esta es una especie de credibilidad y prestigio, porque no hay duda sobre el pasado y el patrimonio cultural, pues son prueba del pasado. Lo que un día fue, tiene su representación en el presente del pueblo, su gente, sus tradiciones, su cultura, su importancia y su esencia como sociedad.

Ahora bien, la preservación es relevante porque transmite distintos valores, mensajes que pueden ser históricos, artísticos, estéticos, políticos, religiosos, sociales, espirituales, naturales y simbólicos, para las generaciones actuales y futuras, además que contribuyen a darle valor a la vida de las personas. Afortunadamente, el patrimonio cultural que tenemos en la actualidad nos remonta al pasado, nos muestra nuestra dilatada historia, nos da un significado importante y nos convierte en alguien con una tradición cultural milenaria y nos hace sentir orgullosos. Es innegable que existimos porque alguna vez fuimos y ese es el valor del pasado, por ello es por lo que recordamos y aún lo vivimos.
Entrando en materia de estudio, Corto Circuito, evento cultural y artístico que surge en la ciudad de Pereira desde hace 15 años, no posee material digital que testifique todos los procesos del mundo cambiante a los que se ha enfrentado. Como se mencionó anteriormente, el recordar es un proceso necesario e inmortalizar los testimonios de fuentes que cuentan con información de primera mano, es clave y la manera de que esta sea trasladada de generación en generación, es dejando registro de ella.

\section{Materiales y métodos}

El método investigativo que se implementó en el presente proyecto es el de investigación-creación, procedimiento que incluye conocimiento, experiencia, intuición, creatividad, innovación, entre otros. Donde se localizaron a los individuos que se han dedicado a realizar el evento Corto Circuito, los gestores de cada institución vinculada; enseguida se planeó y se produjo una entrevista semiestructurada, es decir, mediante un listado de preguntas le dábamos un rumbo, pero a medida que recibíamos las respuestas, surgían nuevas interrogantes para cada uno de ellos, obteniendo así información de primera mano acudiendo a una práctica evocativa del pasado.

No solo fue relevante establecer un proceso comunicativo con los actuales gestores, sino también indagar en los pasados, quienes escucharon la idea de lo que sería Corto Circuito hace 15 años; pues fueron líderes de este, y de esta manera aportar historia, realizar un proceso memorial y simbólico a la investigación; logrando así, relatar cómo ha sido su trascendencia. 
Para la recolección de todos los datos, se partió de la premisa de que cada detalle es de vital importancia y una parte fundamental para la construcción de los contenidos; asimismo, se llegó a la conclusión de que no solo los gestores y fundadores de Corto Circuito son cruciales, sino que también los artistas tienen influencia en la investigación, en virtud de que son el aporte más destacable de este espacio cultural, quienes son la fuente de contenidos y motivación de este. Es por lo anterior que se localizó a algunos de estos para poder conocer su visión, los procesos y rigurosos que experimentan dichos artistas al realizar una exposición, ya que no solo conllevan prácticas investigativas, de creación, sino que también se acarrean relaciones afectivas entre el artista y su obra.

Ahora bien, contamos con una investigación de carácter cualitativo, en vista de que, la observación participativa, fue un procedimiento excepcional para la recolección de información y material, que, además de las entrevistas, se realizó cada mes en el evento durante seis meses consecutivos. El registro audiovisual como diario de campo permitió examinar lo que acaece en él, cómo lo acoge el público y cómo se desenvuelven en su noche de estreno. Una de las insignias de Corto Circuito, son sus tarjetas de invitación, las cuales son un símbolo particular de este y que los asistentes han coleccionado durante años; inequívocamente, forman parte del material de archivo que se busca resaltar en la investigación.

En el proceso de creación de las crónicas, se planteó desde un principio realizar una lista de preguntas, la cuales conta- ban con 10 distintos puntos orientados al trabajo que se realiza y el proceso que se tiene en cuenta a la hora de trabajar en el evento. A partir de esta serie de preguntas, se orientó a los gestores a contarnos cómo es el proceso que se tiene en cuenta al efectuar el evento; también, desde sus perspectivas personales, qué opinan de este espacio cultural y de cómo han logrado mantenerlo todos estos años. En términos estéticos, se ubicó cada gestor en su correspondiente institución, con el propósito de contextualizar a más detalle el espacio cultural del que cada uno forma parte, y realiza la pertinente gestión.

La crónica audiovisual de homenaje a Lucía Molina, se orientó más a un proceso de recordación y de memoria, donde ella cuenta, más que todo, los inicios del evento, y de cómo logró crecer y aportar a la ciudad de Pereira en todos estos años de realización. Nuevamente, se planteó una serie de preguntas base para la entrevista; sin embargo, es una persona que tiene mucho por contar, $y$ a la medida que se le hacían preguntas, surgían más dudas del tema.

En la edición de las crónicas, específicamente en la de los gestores, se pretendió hacer un montaje externo, que contara con cortes claves de entrevista a entrevista, donde cada una de las respuestas de cada uno se complementaran entre sí, y se mantuviera una narración coherente y organizada.

En la crónica de homenaje a Lucía Molina, se destacaron algunas respuestas que ella nos brindó, sobre todo las que contaban con mayor sentimentalismo, 
que siente ella con el evento, validando el hecho de que es un espacio cultural que viene de hace muchos años, y que ha tenido un gran aporte para la ciudad. Por otra parte, la crónica realizada a los artistas, tuvo un enfoque desde una visión externa del evento, a pesar de que son personas que han participado en el evento y han llegado a tener reconocimiento dentro de este; son personajes externos a todo lo que se realiza en Corto Circuito cuando no están exponiendo. Es por esto por lo que se decidió que en esta crónica se destacara la opinión que tienen los artistas pereiranos de este evento, que pretende abrirles las puertas y sacarlos a la luz dentro del marco del arte y la cultura.

\section{Resultados}

Documentar el trayecto durante 15 años del evento Corto Circuito y exponer la visión que tienen de él sus gestores, artistas y público que han participado $\mathrm{y}$ formado parte de este durante 15 años consecutivos, fue el macro objetivo y la necesidad que se necesitaba suplir, pues nadie lo había hecho.

Atestiguar la historia del espacio cultural y artístico de Corto Circuito de la ciudad de Pereira, a partir de una serie de productos audiovisuales que relataran el gran aporte que ha tenido este para con el arte y la cultura de la ciudad de Pereira; puesto que se evidenció una carencia de sentido de pertenencia por la ciudad y los diferentes eventos que se llevan a cabo para esta por parte de la ciudadanía. Es por lo anterior que nació el interés de resaltar y exponer los testimonios de las personas que se encuentran tras bambalinas, quienes hasta el día de hoy hacen posible su ejecución; además, con las piezas construidas conservaremos a través del tiempo su arduo trabajo y, posteriormente, se podrán divulgar para que la ciudadanía se percate de la pertinencia del proyecto y lograr, principalmente, mayor apropiación por este.

Se entiende que el evento cultural, Corto Circuito, se forja a partir del interés de unir a las personas de la ciudad de Pereira por medio de un gusto en común que es el arte, además de la necesidad que se evidencia en ese momento de rescatar la cultura ciudadana; brindándole a esta la oportunidad de asistir a una programación especial donde pudiesen hallar diferentes expresiones del arte, reuniendo así a más de ciento cincuenta mil visitantes durante 15 años de exposiciones. Lo anterior trajo consigo una serie de acontecimientos importantes para la ciudad, tal como mencionó la principal gestora, Lucía Molina: "Las calles del centro eran muy solas, tanto que a la gente le daba miedo caminar por ellas y tuvimos que recurrir a pedir acompañamiento por parte de la policía para hacer los recorridos". Testimonios como el de ella y el de los cinco gestores del evento, relatan cómo se han mantenido a flote durante tantos años consecutivos y siendo ellos quienes más han perseverado para su conservación. Como comentó uno de los gestores, James Llanos: "Otras ciudades han intentado hacer Corto Circuito, pero tan solo en Pereira ha durado tanto".

Se gesta Corto Circuito y se comienza a observar más movimiento en el área céntrica, puesto que se iluminaron las calles, se evidencia la llegada del Me- 
gabús y se inauguran bares en la zona que cumplen su rol de reunir amigos. De esta manera, se debe catalogar como un espacio que aporta al crecimiento cultural, artístico y nocturno de la ciudad de Pereira y aun así, hallamos ausencia de apropiación por parte de la población para con el mismo. Por consiguiente, como investigadoras, encontramos pertinente la propuesta de crear una memoria histórica de este escenario cultural, para que perdure en el tiempo a través de las futuras generaciones. Y como ya se mencionó, crear una evidencia del arduo proceso que acarrea gestionar un evento de tal magnitud, mediante la construcción de piezas audiovisuales que narran su gestación, coordinación y ejecución; puesto que es algo que no se ha realizado anteriormente y serán materiales que homenajearán a todas aquellas personas que aportan su grano de arena para que mes a mes, este tenga sus puertas abiertas para toda la población, también podrán ser expuestos para que nuevas personas no familiarizadas con este, tengan la oportunidad de conocerlo, interesarse y apropiarse de él.

Con lo anterior, se entiende a la ciudad, las calles, como un espacio donde se da un constante intercambio simbólico y mutaciones identitarias en un territorio, hablando propiamente de Pereira, que es bastante prolífico culturalmente. Al caminar por las diferentes calles de la ciudad, si nos regalamos un momento de detenimiento y observación, podríamos reconocer todos estos elementos que se encuentran presentes en un recorrido, a su vez se lograría vivir un momento estático y estético con un toque de memoria.

\section{Discusión}

Participar en el semillero de "Memoria y Región”, nos posibilitó tener un acercamiento íntimo con los autores intelectuales y pioneros del Corto Circuito, permitiendo hacer el registro audiovisual del trayecto de los 15 años del evento, creando contenidos en los cuales se expone la visión que tienen de este, sus gestores, artistas y público que han participado y formado parte de él durante su largo recorrido.

Inicialmente, se evidencia que el evento responde a una necesidad en materia de responsabilidad social y cultural, al ser un espacio a disposición, ante todo, de artistas que ejercen o practican alguna de las diversas expresiones del arte, permitiendo así que los artífices emergentes puedan tener un lugar donde exhibir su derroche de creatividad y, sin duda, darse a conocer públicamente. Ahora bien, con lo anterior, proporcionan una actividad a la ciudadanía de entretenimiento, regocijo y conocimiento.

Asimismo, los fundadores pensaron en una actividad que le pudiese aportar mayor circulación a la parte céntrica de la ciudad y, además, el hecho de poder hacer una caminata relativamente corta alrededor de esta zona y a medida que se realiza, cruzarse con lugares de esparcimiento como bares para compartir entre amigos y se logró a cabalidad.

Teniendo en cuenta lo mencionado anteriormente, se patentiza la pertinencia que posee el Corto Circuito para con el arte, la cultura y la ciudadanía pereirana. Como realizadoras de contenido audio- 
visual, nos asignamos la responsabilidad de inmortalizar el evento a modo de homenaje digital, para que futuras generaciones lo acojan igual o mejor que las actuales y le aporten historia al mismo.

A partir de la observación y participación dentro del evento, se concluye que teniendo en cuenta las fuentes que se contactaron para la recolección de datos y los productos audiovisuales que se realizaron, se constata que Corto Circuito ha brindado a la ciudad de Pereira un gran impacto en lo cultural, artístico y ciudadano, aportando no solo mayor visibilidad a las diferentes expresiones del arte $\mathrm{y}$ a las personas que ejecutan las mismas, sino también a la economía central.

Asimismo, los artistas locales han logrado exponer, mejorar su posición y visibilización con sus trabajos innovadores a partir de su participación en Corto Circuito, al igual que otros artistas nacionales e incluso internacionales que lo ven como una oportunidad única para manifestar su arte y darse a conocer a partir de este, clasificando esa experiencia como única.

Dentro del marco de ciudad, hizo del centro de Pereira un espacio más seguro, más visitado y donde el comercio y el circuito del arte tuvieron mayor acogida. Logrando así, que cada viernes en la noche de Corto Circuito, la ciudad tome vida y se colme de un público de todas las edades para disfrutar de un espacio que reúne un interés en común que es el arte.

\section{Agradecimientos}

El presente trabajo de investigación se realizó bajo la supervisión del docente e investigador Franklyn Molano Gaona, a quien se hace necesario extender nuestro más profundo y sincero agradecimiento por hacer posible la realización de este trabajo; gracias a su paciencia, tiempo y dedicación aplicada para que surgiera de manera exitosa. Gracias por su apoyo, inspiración y su capacidad para guiar nuestras ideas, lo cual fue un aporte invaluable.

Asimismo, le otorgamos un especial agradecimiento a cada una de las personas que conforman Corto Circuito, Escenarios para el Arte, pues son la columna vertebral del trabajo por permitir que este evento de impacto fuese materia de investigación para nosotras. Mil y mil gracias.

\section{Referencias}

Se recurrió a diferentes investigaciones elaboradas por diversos autores con un interés en común, y este ha sido el de dar cuenta y memoria a las artes y la cultura en el contexto de ciudad, a partir de eventos claves que forman parte de la historia artística.

Inicialmente, se encontró un proyecto de investigación relacionado con el arte de la ciudad de Cali, un trabajo que se enfoca en una revisión histórica sobre el contexto artístico local, nacional y latinoamericano, donde Ríos, A. (2013), explica:

Principalmente la necesidad de entender el oficio que aprendí como artista en el medio colombiano, cómo fue su historia y sus vínculos con el arte nacional y Latinoamérica. Entenderlo en el contexto de mi localidad, en sus escuelas de arte y en los procesos que acom- 
pañaron la consolidación de lo que en la gente quedó como un imaginario de ciudad y de cultura emblemático: un periodo y un lugar que la hicieron brillar: la década del setenta y todo lo que devino de la marcada institucionalidad cultural del Museo la Tertulia. (p. 107)

Este trabajo de investigación resalta la importancia de preservar la memoria histórica del arte y la cultura, tiene la necesidad de hablar sobre lo que no se ha dicho, y de demostrar que el arte tiene un recorrido clave en la cultura y en la ciudad.

Por otro lado, se encontró un ensayo que plantea uno de los puntos claves de esta investigación, y es el arte efímero, el arte público, en este se habla de la ciudad entendida como una construcción estética y móvil, como un hecho físico o un escenario de realización artística.

\section{Como explica García, M. (2006):}

El análisis de la ciudad desde el urbanismo, el cuestionamiento a la noción de arte público que establece la historiografía del arte, algunos aportes de la estética contemporánea, así como las herramientas metodológicas del análisis del discurso y la semiótica, serán algunas de las áreas que se intentan convocar para una lectura que integre las prácticas del arte efímero y el espacio urbano. (p. 47)

En este ensayo dice que habitualmente hablamos de la ciudad, y con menor frecuencia de la relación de ella con el arte, con esto también se habla de que la ciudad proyecta la historia de una comunidad y los discursos de la gente; también hace hincapié en la temporalidad de lo efímero en relación con el espacio urbano, y resulta relevante para comprender los intercambios y préstamos que se suceden en la lectura de lo urbano y las prácticas artísticas.

Ahora bien, expandiendo un poco la visión de arte, artista, cultura y ciudad, se halla un escrito elaborado en Tahiche, Las Palmas, España, donde se aborda con gran pertinencia la crisis de la ciudad moderna al convertirse cada vez más en una urbana; y, a su vez, su desvanecimiento del espacio público por la transformación vivida por la modernidad $y$, algo clave, la participación ciudadana apoyada de la mano del artista.

Gómez, F. (2004), afirma:

[...] el artista debe mostrarse sensible hacia los asuntos e intereses de la comunidad, más allá de las características físicas del emplazamiento. Le concierne la noción de lugar entendida como "contenido humano". Amplía, pues, notablemente la contextualidad y enriquece la noción de espacialidad con capas humanas de tensiones políticas, económicas, étnicas, sociológicas, históricas, culturales, psicológicas... Su interés se centra en hacer un arte comprometido con la ciudadanía. (p. 44)

El escrito expone la relevancia que posee velar por la participación ciudadana en los espacios públicos y artísticos, que realmente las diferentes expresiones del arte, mediante su manipulación por el artista pueda generar un cuestionamiento en la persona que lo consume y pueda existir quizá una retroalimentación y apropiación del espacio/obra. 
Por último, es preciso incluir una tesis de grado escrita por una aspirante a la Licenciatura en Artes Visuales de la UTP (Universidad Tecnológica de Pereira), titulada Papel fantasma: del archivo fotográfico al collage contemporáneo como arte público, que nos sumerge de principio a fin en todo el movimiento que se vive al poner un pie fuera de nuestros espacios privados; así es, en las calles, lo urbanístico y las simbologías de la ciudad que forman parte de nuestro patrimonio visual, que se descubren a través de las relaciones con el entorno.

Del cual destacamos que Pulido, M. (2018), expresa:

Si se observan más de cerca los intersticios, y las coreografías diacrónicas que se generan continuamente en distintos puntos de una ciudad, hallamos microrrelatos, microconflictos y microacontecimientos, que se aglutinan como capas de pintura sobre un lienzo en el que ya se ha escrito y sobre el que se va formando tardíamente un palimpsesto de identidades en el que se sobreponen sociedades instantáneas, situaciones contingentes y realidades movedizas. (p. 10)

\section{Anexos}

\section{Anexo 1}

\section{Entrevista semiestructurada}

Formato de preguntas a gestores

1. ¿Qué es Corto Circuito para usted?

2. Describa el papel de su entidad en el Corto Circuito y su cargo. ¿Hace cuánto participa?

3. ¿Cómo es el proceso de selección de los artistas y cómo se coordinan las exposiciones?
4. ¿Cómo es el proceso de montaje?

5. ¿Cómo se promueve cada exposición?

6. ¿Qué finalidad buscan generar con cada exposición?

7. ¿Cuáles son los inconvenientes más comunes a la hora de montar el evento?

8. ¿Cómo se adquiere el presupuesto?

9. ¿Qué intereses personales le gusta implementar en las propuestas?

10. ¿Cuál ha sido la importancia del Corto en Pereira y Colombia?

\section{Anexo 2}

\section{Entrevista semiestructurada}

Formato de preguntas a Lucía Molina

1. ¿De dónde nace la idea de generar un espacio como Corto Circuito para fomentar el arte?

2. ¿Cómo se empezaron a organizar los primeros Corto Circuito?

3. ¿Por qué se decidió que Corto Circuito fuese en diversos lugares?

4. ¿Cómo surge el nombre de Corto Circuito?

5. ¿Cómo fue la organización en cuanto a gestores y locaciones para el primer Corto Circuito?

6. ¿Cuál ha sido la clave para la permanencia de Corto Circuito por estos 15 años?

7. ¿Cómo ha evolucionado Corto Circuito de hace 15 años en comparación con el de ahora?

Anexo 3

Entrevista semiestructurada

Formato de preguntas a artistas

1. ¿Qué es el arte para usted?

2. ¿Qué tipo de arte realiza? 
3. ¿Qué piensa del arte en Pereira y de la diversidad del mismo que hay?

4. ¿Qué piensa de Corto Circuito?

5. ¿Cómo fue su experiencia en Corto Circuito?

6. ¿Qué papel ha jugado el arte en su vida?

7. ¿Cuál es su motivación e inspiración para hacer arte? 